\title{
Lyndon Words versus Inverse Lyndon Words: Queries on Suffixes and Bordered Words
}

\author{
Paola Bonizzoni ${ }^{1}\left(\mathbb{D}\right.$, Clelia De Felice $^{2}$ (D), Rocco Zaccagnino ${ }^{2}$, \\ and Rosalba Zizza ${ }^{2(凶)}$ \\ 1 Dipartimento di Informatica, Sistemistica e Comunicazione, \\ Università degli Studi di Milano - Bicocca, Viale Sarca 336, Milano, Italy \\ bonizzoni@disco.unimib.it \\ 2 Dipartimento di Informatica, Università degli Studi di Salerno, \\ Via Giovanni Paolo II 132, Fisciano (Salerno), Italy \\ \{cdefelice,rzaccagnino,rzizza\}@unisa.it
}

\begin{abstract}
The Lyndon factorization of a word has been extensively studied in different contexts and several variants of it have been proposed. In particular, the canonical inverse Lyndon factorization ICFL, introduced in [5], maintains the main properties of the Lyndon factorization since it can be computed in linear time and it is uniquely determined. In this paper we investigate new properties of this factorization with the purpose of exploring its use in string queries.

As a main result, we prove an upper bound on the length of the longest common extension (or longest common prefix) for two factors of a word $w$. This bound is at most the maximum length of two consecutive factors of $\operatorname{ICFL}(w)$. A tool used in the proof is a property that we state for factors with nonempty borders in $\operatorname{ICFL}(w)$ : a nonempty border of a factor $m_{i}$ cannot be a prefix of the next factor $m_{i+1}$. Another interesting result relates sorting of global suffixes, i.e., suffixes of a word $w$, and sorting of local suffixes, i.e., suffixes of the factors in $\operatorname{ICFL}(w)$.

Finally, given a word $w$ and a factor $x$ of $w$, we prove that their Lyndon factorizations share factors, except for the first and last term of the Lyndon factorization of $x$. This property suggests that, given two words sharing a common overlap, their Lyndon factorizations could be used to capture the common overlap of these two words.
\end{abstract}

Keywords: Lyndon words $\cdot$ Lyndon factorization $\cdot$ Combinatorial algorithms on words

\section{Introduction}

The Lyndon factorization $\operatorname{CFL}(w)$ of a word $w$ is the unique factorization of $w$ into a sequence of Lyndon words in nonincreasing lexicographic ordering. This factorization is one of the most well-known and extensively studied in different 
contexts, from formal languages to algorithmic stringology and string compression. In particular the notion of a Lyndon word has been shown to be useful in theoretical applications, such as the well known proof of the Runs Theorem [2] as well as in string compression analysis. A connection between the Lyndon factorization and the Lempel-Ziv (LZ) factorization has been given in [18], where it is shown that in general the size of the LZ factorization is larger than the size of the Lyndon factorization, and in any case the size of the Lyndon factorization cannot be larger than a factor of 2 with respect to the size of LZ. This result has been further extended in [28] to overlapping LZ factorizations. The Lyndon factorization has recently revealed to be a useful tool also in investigating queries related to suffixes of a word and sorting such suffixes [25] with strong potentialities [26] for string comparison that have not been completely explored and understood. Relations between Lyndon words and the Burrows-Wheeler Transform (BWT) have been discovered first in [11,23] and, more recently, in [19]. The main interest in such a factorization is also due to the fact that it can be efficiently computed. Linear-time algorithms for computing this factorization can be found in $[15,16]$ whereas an $\mathcal{O}(\lg n)$-time parallel algorithm has been proposed in $[1,13]$.

Most recently, variants of the Lyndon factorization have been introduced and investigated with different motivations. In [5], the notion of an inverse Lyndon word (a word which is strictly greater than each of its proper suffixes) has been introduced to define new factorizations, called inverse Lyndon factorizations. An inverse Lyndon factorization has the property that a word is factorized in a sequence of inverse Lyndon words, in an increasing and prefix-order-free lexicographic ordering, where prefix-order-free means that a factor cannot be a prefix of the next one. A word $w$ which is not an inverse Lyndon word may have several inverse Lyndon factorizations but it admits a canonical inverse Lyndon factorization. This special inverse Lyndon factorization has been introduced in [5] and denoted $\operatorname{ICFL}(w)$ because it is the counterpart of the Lyndon factorization $\operatorname{CFL}(w)$ of $w$, when we use (I)inverse words as factors. Indeed, in [5] it has been proved that $\operatorname{ICFL}(w)$ can be computed in linear time and it is uniquely determined for a word $w$.

In this paper we further investigate $\operatorname{ICFL}(w)$. The main results stated here are the following: (1) we find un upper bound on the length of the longest common prefix of two distinct factors in $\operatorname{ICFL}(w)$, namely the maximal length of two consecutive factors in $\operatorname{ICFL}(w)$ (Proposition 6), (2) we are able to relate sorting of global suffixes, i.e., suffixes of the word $w$, and local suffixes, i.e., suffixes of the factors in $\operatorname{ICFL}(w)$ (Lemma 3).

Differently from Lyndon words, inverse Lyndon words may be bordered. As an intermediate result, we show that if a factor $m_{i}$ in $\operatorname{ICFL}(w)$ has a nonempty border, then such a border cannot be inherited by the next factor, since it cannot be the prefix of the next factor $m_{i+1}$ (Proposition 5). This result is proved by a further investigation on the connection between the Lyndon factorization and the canonical inverse Lyndon factorization of a word, given in [5] through the grouping property. Indeed, given a word $w$ which is not an inverse Lyndon word, the factors in $\operatorname{ICFL}(w)$ are obtained by grouping together consecutive factors 
of the anti-Lyndon factorization of $w$ that form a chain for the prefix order (Proposition 7.7 in [5]).

Another natural question is the following.

Given two words having a common overlap, can we use their Lyndon factorizations to capture the similarity of these words?

A partial positive answer to this question is provided here: given a word $w$ and a factor $x$ of $w$, we prove that their Lyndon factorizations share factors, except for the first and last term of the Lyndon factorization of $x$.

For the detailed proofs of the results in this paper we refer the reader to [6].

\section{Words, Lyndon Words and the Lyndon Factorization}

Throughout this paper we follow $[4,10,20,22,27]$ for the notations. We fix the finite non-empty (totally ordered) alphabet $\Sigma$. We denote by $\Sigma^{*}$ the free monoid generated by $\Sigma$ and we set $\Sigma^{+}=\Sigma^{*} \backslash 1$, where 1 is the empty word. For a word $w \in \Sigma^{*}$, we denote by $|w|$ its length. A word $x \in \Sigma^{*}$ is a factor of $w \in \Sigma^{*}$ if there are $u_{1}, u_{2} \in \Sigma^{*}$ such that $w=u_{1} x u_{2}$. If $u_{1}=1$ (resp. $u_{2}=1$ ), then $x$ is a prefix (resp. suffix) of $w$. A factor (resp. prefix, suffix) $x$ of $w$ is proper if $x \neq w$. Given a language $L \subseteq A^{*}$, we denote by $\operatorname{Pref}(L)$ the set of all prefixes of its elements. Two words $x, y$ are incomparable for the prefix order if neither $x$ is a prefix of $y$ nor $y$ is a prefix of $x$. Otherwise, $x, y$ are comparable for the prefix order. We write $x \leq_{p} y$ if $x$ is a prefix of $y$ and $x \geq_{p} y$ if $y$ is a prefix of $x$. We recall that, given a nonempty word $w$, a border of $w$ is a word which is both a proper prefix and a suffix of $w[12]$. The longest proper prefix of $w$ which is a suffix of $w$ is also called the border of $w[12,22]$. A word $w \in \Sigma^{+}$is bordered if it has a nonempty border. Otherwise, $w$ is unbordered. A nonempty word $w$ is primitive if $w=x^{k}$ implies $k=1$. An unbordered word is primitive. A sesquipower of a word $x$ is a word $w=x^{n} p$ where $p$ is a proper prefix of $x$ and $n \geq 1$.

The lexicographic (or alphabetic order) $\prec$ on $\left(\Sigma^{*},<\right.$ ) is defined by setting $x \prec y$ if $x$ is a proper prefix of $y$, or $x=r a s, y=r b t, a<b$, for $a, b \in \Sigma$ and $r, s, t \in \Sigma^{*}$. For two nonempty words $x, y$, we write $x \ll y$ if $x \prec y$ and $x$ is not a proper prefix of $y$ [3]. We also write $y \succ x$ if $x \prec y$. Two words $x, y$ are called conjugate if there exist words $u, v$ such that $x=u v, y=v u$. The conjugacy relation is an equivalence relation. A conjugacy class is a class of this equivalence relation. A Lyndon word $w \in \Sigma^{+}$is a word which is primitive and the smallest one in its conjugacy class for the lexicographic order. A class of conjugacy is also called a necklace and often identified with the minimal word for the lexicographic order in it. Thus, a nonempty word is a necklace if and only if it is a power of a Lyndon word. A prenecklace is a prefix of a necklace, hence any nonempty prenecklace $w$ has the form $w=(u v)^{k} u$, where $u v$ is a Lyndon word, $u \in \Sigma^{*}, v \in \Sigma^{+}, k \geq 1$, that is, $w$ is a sesquipower of a Lyndon word $u v$. A characterization of the structure of the prefixes of the Lyndon words is given in [15]. It states that a word is a nonempty prefix of a Lyndon word if and only if it is a sesquipower of a Lyndon word distinct of the maximal letter. 
It is known that each Lyndon word $w$ is unbordered. Moreover, a word $w \in$ $\Sigma^{+}$is a Lyndon word if and only if $w \prec s$, for each nonempty proper suffix $s$ of $w$. Different characterizations and variations of Lyndon words are given $[3,14,21]$. In the following $L=L_{\left(\Sigma^{*},<\right)}$ will be the set of Lyndon words, totally ordered by the relation $\prec$ on $\left(\Sigma^{*},<\right)$. We know that any word $w \in \Sigma^{+}$can be written in a unique way as a nonincreasing product $w=\ell_{1} \ell_{2} \cdots \ell_{h}$ of Lyndon words, i.e., in the form $w=\ell_{1} \ell_{2} \cdots \ell_{h}$, with $\ell_{j} \in L$ and $\ell_{1} \succeq \ell_{2} \succeq \ldots \succeq \ell_{h}$ [9]. The sequence $\operatorname{CFL}(w)=\left(\ell_{1}, \ldots, \ell_{h}\right)$ is called the Lyndon decomposition (or Lyndon factorization) of $w$. Uniqueness of the above factorization is proved in [15] and allows us to state a recursive definition of $\operatorname{CFL}(w)$, for a nonempty word $w$. Precisely, if $w$ is not a Lyndon word, then $\operatorname{CFL}(w)=\left(\ell_{1}, \ell_{1}^{\prime}, \ldots, \ell_{h}^{\prime}\right)$, where $\left(\ell_{1}^{\prime}, \ldots, \ell_{h}^{\prime}\right)=\operatorname{CFL}\left(w^{\prime}\right), w=\ell_{1} w^{\prime}$ and $\ell_{1}$ is the longest prefix of $w$ which is a Lyndon word. Sometimes we need to emphasize consecutive equal factors in CFL. We write $\operatorname{CFL}(w)=\left(\ell_{1}^{n_{1}}, \ldots, \ell_{r}^{n_{r}}\right)$ to denote a tuple of $n_{1}+\ldots+n_{r}$ Lyndon words, where $r>0, n_{1}, \ldots, n_{r} \geq 1$. Precisely $\ell_{1} \succ \ldots \succ \ell_{r}$ are Lyndon words, also named Lyndon factors of $w$. There is a linear time algorithm to compute the pair $\left(\ell_{1}, n_{1}\right)$ and thus, by iteration, the Lyndon factorization of $w[16,22]$. Linear time algorithms may also be found in [15] and in the more recent paper [17].

\section{Anti-Lyndon Words, Inverse Lyndon Words and Anti-prenecklaces}

The inverse lexicographic or inverse alphabetic order on $\left(\Sigma^{*},<\right)$, denoted $\prec_{\text {in }}$, is the lexicographic order on $\left(\Sigma^{*},<_{i n}\right)$. Here $<_{i n}$ means that the order of the alphabet is reversed, that is $b<_{\text {in }} a \Leftrightarrow a<b$, for all $a, b \in \Sigma$. We denote by $L_{i n}=L_{\left(\Sigma^{*},<_{i n}\right)}$ the set of the Lyndon words on $\Sigma^{*}$ with respect to the inverse lexicographic order. A word $w \in L_{i n}$ will be named an anti-Lyndon word. Correspondingly, an anti-prenecklace will be a prefix of an anti-necklace, which in turn will be a necklace with respect to the inverse lexicographic order. We have that a word $w \in \Sigma^{+}$is in $L_{i n}$ if and only if $w$ is primitive and $w \succ v u$, for each $u, v \in \Sigma^{+}$such that $w=u v$. Alternatively, a word $w \in \Sigma^{+}$is in $L_{i n}$ if and only if $w$ is unbordered and $w \succ v$, for each proper nonempty suffix $v$. We denote by $\mathrm{CFL}_{i n}(w)$ the Lyndon factorization of $w$ with respect to the inverse order $<_{i n}$. The following definition plays a fundamental role in our results.

Definition 1. A word $w \in \Sigma^{+}$is an inverse Lyndon word if $s \prec w$, for each nonempty proper suffix $s$ of $w$.

It is easy to see that $a, b, a a a a a, b b b a, b a a a b, b b a b a$ and bbababbaa are inverse Lyndon words on $\{a, b\}$, with $a<b$. On the contrary, aaba is not an inverse Lyndon word since $a a b a \prec b a$. Moreover, baaab is not an anti-Lyndon word since it is bordered. In [5] it has been proved that a nonempty word is an antiLyndon word if and only if it is an unbordered inverse Lyndon word. Finally, the set of the inverse Lyndon words coincides with the set of the anti-prenecklaces, hence any nonempty prefix of an inverse Lyndon word is still an inverse Lyndon word [5]. 


\section{A Canonical Inverse Lyndon Factorization: $\operatorname{ICFL}(w)$}

For the material in this section see [5]. An inverse Lyndon factorization of a word $w \in \Sigma^{+}$is a sequence $\left(m_{1}, \ldots, m_{k}\right)$ of inverse Lyndon words such that $m_{1} \cdots m_{k}=w$ and $m_{i} \ll m_{i+1}, 1 \leq i \leq k-1$. A word may have different inverse Lyndon factorizations (see Example 2) but it has a unique canonical inverse Lyndon factorization, denoted $\operatorname{ICFL}(w)$. If $w$ is an inverse Lyndon word, then $\operatorname{ICFL}(w)=w$. Otherwise, $\operatorname{ICFL}(w)$ is recursively defined. The first factor of $\operatorname{ICFL}(w)$ is obtained by a special factorization of the shortest nonempty prefix $z$ of $w$ such that $z$ is not an inverse Lyndon word.

Definition 2 [5]. Let $w \in \Sigma^{+}$, let $p$ be an inverse Lyndon word which is a nonempty proper prefix of $w=p v$. The bounded right extension $\bar{p}$ of $p$ (relatively to $w)$, if it exists, is a nonempty prefix of $v$ such that:

(1) $\bar{p}$ is an inverse Lyndon word,

(2) $p z^{\prime}$ is an inverse Lyndon word, for each proper nonempty prefix $z^{\prime}$ of $\bar{p}$,

(3) $p \bar{p}$ is not an inverse Lyndon word,

(4) $p \ll \bar{p}$.

Moreover, we set $\operatorname{Pref}_{b r e}(w)=\{(p, \bar{p}) \mid p$ is an inverse Lyndon word which is a nonempty proper prefix of $w$.

It has been proved that $\operatorname{Pref}_{b r e}(w)$ is empty if and only if $w$ is an inverse Lyndon word (Proposition 4.2 in [5]). If $w$ is not an inverse Lyndon word, then $\operatorname{Pref}_{b r e}(w)$ contains only one pair and the description of this pair is given below (Propositions 4.1 and 4.3 in [5]).

Proposition 1. Let $w \in \Sigma^{+}$be a word which is not an inverse Lyndon word. Let $z$ be the shortest nonempty prefix of $w$ which is not an inverse Lyndon word. Then,

(1) $z=p \bar{p}$, with $(p, \bar{p}) \in \operatorname{Pref}_{\text {bre }}(w)$.

(2) $p=$ ras and $\bar{p}=r b$, where $r, s \in \Sigma^{*}, a, b \in \Sigma$ and $r$ is the shortest prefix of $p \bar{p}$ such that $p \bar{p}=$ rasrb, with $a<b$.

Example 1. Let $\Sigma=\{a, b\}$ with $a<b$. Let us consider $w=$ babaaabb and the prefixes $p_{1}=b a b$ and $p_{2}=b a b a a a$ of $w$. First, $w$ is not an inverse Lyndon word. Thus, Pref bre $(w)$ contains only one pair. Moreover each proper nonempty prefix of $w$ is an inverse Lyndon word. By item (1) in Proposition 1, we have $w=p \bar{p}$. By item (2) in Proposition 1, the bounded right extension of $p_{1}=b a b$ does not exist (we should have $\overline{p_{1}}=a a a b b$ in contradiction with $p_{1} \ll \overline{p_{1}}$ ). Since $w$ starts with $b$, the shortest common prefix $r$ of $p$ and $\bar{p}$ has a positive length. Indeed, $p=p_{2}=$ babaaa and $\bar{p}=\overline{p_{2}}=b b$.

The canonical inverse Lyndon factorization has been also recursively defined. 
Definition 3. Let $w \in \Sigma^{+}$.

(Basis Step) If $w$ is an inverse Lyndon word, then $\operatorname{ICFL}(w)=(w)$.

(Recursive Step) If $w$ is not an inverse Lyndon word, let $(p, \bar{p}) \in \operatorname{Pref}_{\text {bre }}(w)$ and let $v \in \Sigma^{*}$ such that $w=p v$. Let $\operatorname{ICFL}(v)=\left(m_{1}^{\prime}, \ldots, m_{k}^{\prime}\right)$ and let $r, s \in \Sigma^{*}$, $a, b \in \Sigma$ such that $p=$ ras, $\bar{p}=r b$ with $a<b$.

$$
\operatorname{ICFL}(w)= \begin{cases}(p, \operatorname{ICFL}(v)) & \text { if } \bar{p}=r b \leq_{p} m_{1}^{\prime} \\ \left(p m_{1}^{\prime}, m_{2}^{\prime}, \ldots, m_{k}^{\prime}\right) & \text { if } m_{1}^{\prime} \leq_{p} r\end{cases}
$$

Example 2 [5]. Let $\Sigma=\{a, b, c, d\}$ with $a<b<c<d, w=$ dabadabdabdadac. We have $\mathrm{CFL}_{i n}(w)=($ daba,dab,dab,dadac $), \operatorname{ICFL}(w)=($ daba, dabdab, dadac $)$. Another inverse Lyndon factorizations of $w$ is (dabadab,dabda,dac). Consider $z=d a b d a d a c d d b d c$. It is easy to see that (dab, dadacd, db, dc), (dabda, $d a c, d d b d c),(d a b, d a d a c, d d b d c)$ are all inverse Lyndon factorizations of $z$. The first factorization has four factors whereas the others have three factors. Moreover $\operatorname{ICFL}(z)=\mathrm{CFL}_{i n}(z)=(d a b, d a d a c, d d b d c)$.

\section{Groupings and Borders}

Let $w \in \Sigma^{+}$be a word which is not an inverse Lyndon word, let $\operatorname{ICFL}(w)=$ $\left(m_{1}, \ldots, m_{k}\right)$. The aim of this section is to state that any nonempty border of $m_{i}$ is not a prefix of $m_{i+1}, 1 \leq i \leq k-1$ (Proposition 5). The proof of this result is strongly based on a property of $\operatorname{ICFL}(w)$, proved in [5] and defined through the notion of groupings of $\mathrm{CFL}_{i n}(w)$.

Let $\mathrm{CFL}_{i n}(w)=\left(\ell_{1}, \ldots, \ell_{h}\right)$, where $\ell_{1} \succeq_{\text {in }} \ell_{2} \succeq_{i n} \ldots \succeq_{\text {in }} \ell_{h}$. Consider the partial order $\geq_{p}$, where $x \geq_{p} y$ if $y$ is a prefix of $x$. Recall that a chain is a set of a pairwise comparable elements. We say that a chain is maximal if it is not strictly contained in any other chain. A non-increasing (maximal) chain in $\mathrm{CFL}_{i n}(w)$ is the sequence corresponding to a (maximal) chain in the multiset $\left\{\ell_{1}, \ldots, \ell_{h}\right\}$ with respect to $\geq_{p}$. We denote by $\mathcal{P} \mathcal{M C}$ a non-increasing maximal chain in $\operatorname{CFL}_{i n}(w)$. Looking at the definition of the (inverse) lexicographic order, it is easy to see that a $\mathcal{P} \mathcal{M C}$ is a sequence of consecutive factors in $\mathrm{CFL}_{i n}(w)$. Moreover $\mathrm{CFL}_{i n}(w)$ is the concatenation of its $\mathcal{P} \mathcal{M C}$. Formally, if $\mathcal{C}$ is a $\mathcal{P} \mathcal{M C}$ in $\mathrm{CFL}_{i n}(w)$, then there are indexes $r, s$ with $1 \leq r<s \leq h$ such that $\mathcal{C}=\left(\ell_{r}, \ldots, \ell_{s}\right)$, with $\ell_{r} \geq_{p} \ell_{r+1} \geq_{p} \ldots \geq_{p} \ell_{s}$, and $\ell_{r-1} \geq_{p} \ell_{r}$ if $r>1, \ell_{s} \geq_{p} \ell_{s+1}$ if $s<h$.

Example 3 [5]. Let $\Sigma=\{a, b, c, d\}$ with $a<b<c<d, w=$ dabadabdabdadac. In Example 2, we observed that $\mathrm{CFL}_{i n}(w)=(d a b a, d a b, d a b, d a d a c)$. This sequence has two $\mathcal{P} \mathcal{M C}$, namely $(d a b a, d a b, d a b),(d a d a c)$. Let $z=$ dabdadacddbdc. Then $\mathrm{CFL}_{i n}(z)=(d a b, d a d a c, d d b d c)$ has three $\mathcal{P} \mathcal{M C}:(d a b),($ dadac $),(d d b d c)$.

A grouping of $\mathrm{CFL}_{i n}(w)$ is an inverse Lyndon factorization $\left(m_{1}, \ldots, m_{k}\right)$ of $w$ such that any factor is a product of consecutive factors in a $\mathcal{P} \mathcal{M C}$ of $\mathrm{CFL}_{i n}(w)$. $\operatorname{ICFL}(w)$ is always a grouping of $\mathrm{CFL}_{i n}(w)$ but, as showed below, it is not always its unique grouping. 
Example 4 [5]. Let $\Sigma=\{a, b, c, d\}, a<b<c<d$, and $w=$ dabadabdabdadac. We have $\mathrm{CFL}_{i n}(w)=($ daba, dab, dab,dadac $), \operatorname{ICFL}(w)=($ daba, dabdab, dadac $)$ (see Example 2). $\operatorname{ICFL}(w)$ is a grouping of $\mathrm{CFL}_{i n}(w)$ but (dabadab, dabda, dac) is not a grouping. Next, let $y=$ dabadabdabdabdadac. We have $\mathrm{CFL}_{i n}(y)=$ $(d a b a, d a b, d a b, d a b, d a d a c)$ and $\operatorname{ICFL}(w)=\left(d a b a,(d a b)^{3}, d a d a c\right)$. The inverse Lyndon factorization (dabadab, $(d a b)^{2}$, dadac) is another grouping of $\mathrm{CFL}_{i n}(y)$.

The proof of Proposition 5 is organized as follows. We firstly state that any nonempty border $x$ of a non-increasing chain in $\mathrm{CFL}_{i n}(w)$ cannot cut any $\ell_{i}$ and admits a shortest border.

Proposition 2. Let $w \in \Sigma^{+}$, let $\mathrm{CFL}_{i n}(w)=\left(\ell_{1}, \ldots, \ell_{h}\right)$ and let $\left(\ell_{r}, \ldots, \ell_{s}\right)$, $1 \leq r<s \leq h$, be a non-increasing chain in $\mathrm{CFL}_{i n}(w)$. For any nonempty border $x$ of $y=\ell_{r} \cdots \ell_{s}$ there is $t, r \leq t<s$, such that $x=\ell_{t+1} \cdots \ell_{s}$. Consequently, $\ell_{s}$ is a nonempty border of any other nonempty border of $\ell_{r} \cdots \ell_{s}$.

The next step is to prove that $p$ in the pair $(p, \bar{p}) \in \operatorname{Pref}_{b r e}(w)$ has a groupinglike property. Indeed we show that $p$ is always a product of consecutive factors in a $\mathcal{P} \mathcal{M C}$ of $\mathrm{CFL}_{i n}(w)$. Thus, thanks to Proposition $2, p$ has a shortest border. This shortest border determines the relation $p \ll \bar{p}$.

Proposition 3. Let $w \in \Sigma^{+}$be a word which is not an inverse Lyndon word, let $(p, \bar{p}) \in \operatorname{Pref}_{b r e}(w)$ and let $\operatorname{ICFL}(w)=\left(m_{1}, \ldots, m_{k}\right)$. Let $\mathrm{CFL}_{i n}(w)=$ $\left(\ell_{1}^{n_{1}}, \ldots, \ell_{h}^{n_{h}}\right)$, with $h>0, n_{1}, \ldots, n_{h} \geq 1$ and let $\left(\ell_{1}^{n_{1}}, \ldots, \ell_{q}^{n_{q}}\right)$ be a $\mathcal{P} \mathcal{M C}$ in $\mathrm{CFL}_{\text {in }}(w), 1 \leq q \leq h$. Then the following properties hold.

(1) $p=\ell_{1}^{n_{1}} \cdots \ell_{g}^{n_{g}}$, for some $g, 1 \leq g \leq q$.

(2) $\ell_{g}=u_{g} v_{g}=u_{g} a_{g} v_{g}^{\prime}, \bar{p}=u_{g} b, a_{g}<b$.

Now, we can state that, for each nonempty border $z$ of $p=$ ras, we have that $z$ and $\bar{p}=r b$ are incomparable for the prefix order. We use the same notations as in Propositions 2, 3. The word $\bar{p}$ cannot be a prefix of $z$ because $\bar{p}$ is not a prefix of $p$. Thus $z$ should be a prefix of $\bar{p}$. By Proposition 2, the shortest border $\ell_{g}=u_{g} a_{g} v_{g}^{\prime}$ of $p$ should be a prefix of $z$, thus of $\bar{p}=u_{g} b, a_{g}<b$, a contradiction.

Proposition 4. Let $w \in \Sigma^{+}$be a word which is not an inverse Lyndon word and let $(p, \bar{p}) \in \operatorname{Pref}_{\text {bre }}(w)$. For each nonempty border $z$ of $p$, one has that $z$ and $\bar{p}$ are incomparable for the prefix order.

Finally, we can explicitly prove, by induction on $|w|$, that if $z$ is a nonempty border of $m_{1}$, then $z$ is not a prefix of $m_{2}$. We use the recursive definition of $\operatorname{ICFL}(w)$, with the same notations as in Definition 3, and a proof by induction. We distinguish the two cases $m_{1}=p$ and $m_{1}=p m_{1}^{\prime}$. In the first case, $\bar{p}$ is a prefix of $m_{1}^{\prime}=m_{2}$. Thus, if $z$ were a prefix of $m_{2}$, we would be in contradiction with Proposition 4. In the second case, we have $m_{2}=m_{2}^{\prime}$ and again two cases: $|z| \geq\left|m_{1}^{\prime}\right|$ or $|z|<\left|m_{1}^{\prime}\right|$. If $z$ were a prefix of $m_{2}$ with $|z| \geq\left|m_{1}^{\prime}\right|, m_{1}^{\prime}$ would be a prefix of $m_{2}^{\prime}$ in contradiction with $m_{1}^{\prime} \ll m_{2}^{\prime}$. If $z$ were a prefix of $m_{2}$ with $|z|<\left|m_{1}^{\prime}\right|$, $z$ would be a border of $m_{1}^{\prime}$, in contradiction with the induction hypothesis. Then, again by induction on $|w|$, we extend this argument to prove the general result stated below. 
Proposition 5. Let $w \in \Sigma^{+}$be a word which is not an inverse Lyndon word and let $\operatorname{ICFL}(w)=\left(m_{1}, \ldots, m_{k}\right)$. If $z$ is a nonempty border of $m_{i}$, then $z$ is not a prefix of $m_{i+1}, 1 \leq i \leq k-1$.

\section{A Bound on the Length of the Longest Common Prefix}

Given a word $w$ and two factors $x, y$ of $w$, we denote by $\operatorname{lcp}(x, y)$ the longest common prefix of $x, y$ and we set $\operatorname{LCP}(x, y)=|\operatorname{lcp}(x, y)|$. Proposition 5 in the previous section is extremely useful to obtain a bound on the length of the longest common prefix of two factors of a word $w$, when $w$ is not an inverse Lyndon word (Proposition 6). Precisely, we state that $\operatorname{LCP}(x, y)$ is at most the maximum length of two consecutive factors in $\operatorname{ICFL}(w)$. As a direct corollary, we obtain the same bound for $\operatorname{LCP}(x, y)$, when $x, y$ are suffixes of $w$ [6].

We also follow the notations used in $[5,24,25]$. Let $w, x, u, v \in \Sigma^{*}$, and let $x$ be a nonempty factor of $w=u x v$. Let first $(x)$ and last $(x)$ denote the position of the first and the last symbol of $x$ in $w$, respectively. If $w=a_{1} \cdots a_{n}, a_{i} \in \Sigma$, $1 \leq i \leq j \leq n$, then we also set $w[i, j]=a_{i} \cdots a_{j}$. A local suffix of $w$ is a suffix of a factor of $w$, specifically $\operatorname{suf}_{x}(i)=w[i$, last $(x)]$ denotes the local suffix of $w$ at the position $i$ with respect to $x, i \geq f \operatorname{irst}(x)$. The corresponding global suffix $s u f_{x}(i) v$ of $w$ at the position $i$ is denoted by $s u f_{w}(i)=w[i, \operatorname{last}(w)]$ (or simply $\operatorname{suf}(i)$ when it is understood). We say that $s u f_{x}(i) v$ is associated with $s u f_{x}(i)$.

When we consider $\operatorname{ICFL}(w)=\left(m_{1}, \ldots, m_{k}\right)$, given a factor $m_{j}$ of $\operatorname{ICFL}(w)$ we have that a local suffix $x$ of $m_{j}$ is a suffix of $m_{j}$ and the associated global suffix $x_{w}$ of $w$ is $x \cdot m_{j+1} \ldots m_{k}$. The following lemmas are crucial for proving our upper bound. Lemma 1 shows that, given two local suffixes $x$ and $y$ of the same factor $m_{i-1}$, then the longest common prefix of the associated global suffixes is the longest common prefix between $x r$ and $y r$. Here $r$ is the longest common prefix between $m_{i-1}$ and $m_{i}$. Lemma 2 handles the case of local suffixes $x$ and $y$ of different factors. In this case the result leads to a bound on $\operatorname{LCP}\left(x_{w}, y_{w}\right)$.

Lemma 1. Let $w \in \Sigma^{+}$be a word which is not an inverse Lyndon word. Let $\operatorname{ICFL}(w)=\left(m_{1}, \ldots, m_{k}\right)$. Let $r, s, t \in \Sigma^{*}, a, b \in \Sigma$ be such that $m_{i-1}=$ ras, $m_{i}=r b t, a<b, 1<i \leq k$. If $x, y$ are nonempty suffixes of $m_{i-1}$, then $\operatorname{lcp}\left(x_{w}, y_{w}\right)=\operatorname{lcp}(x r, y r)$.

Lemma 2. Let $w \in \Sigma^{+}$be a word which is not an inverse Lyndon word and let $\operatorname{ICFL}(w)=\left(m_{1}, \ldots, m_{k}\right)$. Let $i, j$ be integers such that $1<i<j \leq k$. If $x$ is a nonempty suffix of $m_{i-1}$ and $y$ is a nonempty suffix of $m_{j-1}$, then $\operatorname{lcp}\left(x_{w}, y_{w}\right)$ is a prefix of $y_{j}$.

Let $w \in \Sigma^{+}$be a word which is not an inverse Lyndon word and let $\operatorname{ICFL}(w)=\left(m_{1}, \ldots, m_{k}\right)$. We set $\mathcal{M}=\max \left\{\left|m_{i} m_{i+1}\right| \mid 1 \leq i<k\right\}$. As a main consequence of the previous lemmas, we state that $\mathcal{M}$ is an upper bound on $\operatorname{LCP}(u, v)$, where $u, v$ are factors of $w$.

Proposition 6. Let $w \in \Sigma^{+}$be a word which is not an inverse Lyndon word and let $\operatorname{ICFL}(w)=\left(m_{1}, \ldots, m_{k}\right)$. For each nonempty proper factors $u, v$ of $w$, we have $\operatorname{LCP}(u, v)=|\operatorname{lcp}(u, v)| \leq \mathcal{M}$. 
Observe that Lemmas 1 and 2 could lead to a more specialized version of the compatibility property, proved in $[5,24,25]$, which relates sorting local suffixes of a concatenation of factors to sorting the corresponding global suffixes (see Theorem 1). Indeed the above mentioned lemmas could be applied to sort suffixes of a word by sorting factors of $w$ of bounded size.

We recall that the sorting of the nonempty local suffixes of $w$ with respect to a nonempty factor $x$ is compatible with the sorting of the corresponding nonempty global suffixes of $w$ if for all $i, j$ with $\operatorname{first}(x) \leq i<j \leq \operatorname{last}(x), \operatorname{su} f_{x}(i) \prec$ $\operatorname{suf} f_{x}(j) \Longleftrightarrow \operatorname{suf}(i) \prec s u f(j)$.

Theorem 1 [24,25]. Let $w \in \Sigma^{+}$and let $\operatorname{CFL}(w)=\left(\ell_{1}, \ldots, \ell_{h}\right)$ be its Lyndon factorization. Then, for any $r, s, 1 \leq r \leq s \leq h$, the sorting of the nonempty local suffixes of $w$ with respect to $x=\ell_{r} \cdots \ell_{s}$ is compatible with the sorting of the corresponding nonempty global suffixes of $w$.

Lemma 3 states a property similar to the compatibility property when we deal with $\operatorname{ICFL}(w)$. Shortly speaking, consider $\operatorname{ICFL}(w)=\left(m_{1}, m_{2}, \ldots, m_{k}\right)$ and take two indexes $j_{1}, j_{2}$ both contained in $x=m_{r} m_{r+1} \cdots m_{s}, 1 \leq r<s \leq k$. Consider the local suffixes starting from $j_{1}, j_{2}$ and let us compare them with respect to $\prec$. If $s u f_{x}\left(j_{1}\right) \prec s u f_{x}\left(j_{2}\right)$, then two cases are possible: $s u f_{x}\left(j_{1}\right) \ll s u f_{x}\left(j_{2}\right)$ or $s u f_{x}\left(j_{1}\right) \in \operatorname{Pref}\left(s u f_{x}\left(j_{2}\right)\right)$. In the first case obviously $\operatorname{suf}\left(j_{1}\right) \ll \operatorname{suf}\left(j_{2}\right)$. Lemma below covers both the cases.

Lemma 3. Let $w \in \Sigma^{+}$be a word which is not an inverse Lyndon word and let $\operatorname{ICFL}(w)=\left(m_{1}, \ldots, m_{k}\right)$. Let $x=m_{i} m_{i+1} \cdots m_{h}$ with $1 \leq i<h \leq k$. Assume that suf $f_{x}\left(j_{1}\right) \prec \operatorname{suf}_{x}\left(j_{2}\right)$, where first $(x) \leq j_{1} \leq \operatorname{last}(\bar{x})$, first $(\bar{x}) \leq$ $j_{2} \leq \operatorname{last}(x), j_{1} \neq j_{2}$. If suf $f_{x}\left(j_{1}\right)$ is a proper prefix of $s u f_{x}\left(j_{2}\right)$ and $h<k$ then $\operatorname{suf}\left(j_{2}\right) \prec \operatorname{suf}\left(j_{1}\right)$, otherwise suf $\left(j_{1}\right) \prec \operatorname{suf}\left(j_{2}\right)$.

Example 5. Let $w=a^{12} b b a b \in\{a, b\}^{+}$with $a<b$. We have $\operatorname{ICFL}(w)=$ $\left(m_{1}, m_{2}\right)=\left(a^{12}, b b a b\right)$. Let $x=m_{1}=a^{12}$. Consider $s u f_{x}(4)=a^{9}$ and $\operatorname{suf}_{x}(12)=a$. We have $\operatorname{suf}_{x}(12)=a \prec a^{9}=s u f_{x}(4)$. We are in the first case of Lemma 3 and then $\operatorname{suf}(4)=a^{9} b b a b \prec a b b a b=\operatorname{suf}(12)$.

Example 6. Let $w=$ dabadabdabdadac $\in\{a, b, c, d\}^{+}$with $a<b<c<d$. We have $\operatorname{ICFL}(w)=\left(m_{1}, m_{2}, m_{3}\right)=(d a b a, d a b d a b, d a d a c)$. Let $x=m_{2}$. Consider $s u f_{m_{2}}(8)=d a b$ and $s u f_{m_{2}}(5)=d a b d a b$. We have $s u f_{m_{2}}(8)=d a b \prec$ $s u f_{m_{2}}(5)=d a b d a b=(d a b)^{2}$. We are in the first case of Lemma 3 and then $\operatorname{suf}(5)=$ dabdabdadac $\prec \operatorname{suf}(8)=$ dabdadc. Consider now $s u f_{m_{2}}(9)=a b \prec$ $s u f_{m_{2}}(8)=d a b$. Since $s u f_{m_{2}}(9)$ is not a proper prefix of $\left.s u f_{m_{2}}(8)\right)$, we are in the second case of Lemma 3 and we have $s u f(9)=$ abdadac $\prec s u f(8)=$ dabdadac.

\section{Lyndon Factorizations of Factors of a Word and Overlapping Factors}

Let $w \in \Sigma^{+}$be a word and let $\operatorname{CFL}(w)=\left(\ell_{1}, \ldots, \ell_{k}\right)$ be its Lyndon factorization, $k \geq 1$. Let $x$ be a proper factor (resp. prefix, suffix) of $w$. We say that $x$ is a 
simple factor of $w$ if, for each occurrence of $x$ as a factor of $w$, there is $j$, with $1 \leq j \leq k$, such that $x$ is a factor of $\ell_{j}$. Informally speaking, every occurrence of $x$ needs to be within some $\ell_{j}$. We say that $x$ is a simple prefix (resp. suffix) of $w$ if $x$ is a proper prefix (resp. suffix) of $\ell_{1}\left(\operatorname{resp} \ell_{k}\right)$. In this section we compare the Lyndon factorization of $w$ and that of its non-simple factors. Lemma 4 handles a trivial case: if $x=\ell_{i} \ell_{i+1} \cdots \ell_{j}$ is a concatenation of consecutive factors of $\operatorname{CFL}(w)$, then $\operatorname{CFL}(x)$ is the sequence $\left(\ell_{i}, \ell_{i+1}, \ldots, \ell_{j}\right)$.

Lemma 4. Let $w \in \Sigma^{+}$be a word and let $\operatorname{CFL}(w)=\left(\ell_{1}, \ldots, \ell_{k}\right)$ be its Lyndon factorization. For any $i, j$, with $1 \leq i \leq j \leq k$, one has $\operatorname{CFL}\left(\ell_{i} \ell_{i+1} \cdots \ell_{j}\right)=$ $\left(\ell_{i}, \ell_{i+1}, \ldots, \ell_{j}\right)$

If $x$ is a non-simple factor of $w$ and $x$ does not satisfy the hypotheses of Lemma 4 , then there are $i, j$ with $1 \leq i<j \leq k$, a suffix $\ell_{i}^{\prime \prime}$ of $\ell_{i}$ and a prefix $\ell_{j}^{\prime}$ of $\ell_{j}$, with $\ell_{i}^{\prime \prime} \ell_{j}^{\prime} \neq 1$, such that $x=\ell_{i}^{\prime \prime} \ell_{i+1} \cdots \ell_{j-1} \ell_{j}^{\prime}$, where it is understood that if $j=i+1$, then $\ell_{i+1}, \cdots, \ell_{j-1}=1$ and $\ell_{i}^{\prime \prime} \neq 1, \ell_{j}^{\prime} \neq 1, \ell_{i}^{\prime \prime} \ell_{j}^{\prime} \neq \ell_{i} \ell_{j}$. We say that the sequence $\ell_{i}^{\prime \prime}, \ell_{i+1}, \ldots, \ell_{j-1}, \ell_{j}^{\prime}$ is associated with $x$. The following result gives relations between $\operatorname{CFL}(x)$ and $\operatorname{CFL}(w)$.

Lemma 5. Let $w \in \Sigma^{+}$be a word and let $\operatorname{CFL}(w)=\left(\ell_{1}, \ldots, \ell_{k}\right)$ be its Lyndon factorization. Let $x$ be a non-simple factor of $w$ such that $x$ does not satisfy the hypotheses of Lemma 4 and let $\ell_{i}^{\prime \prime}, \ell_{i+1}, \ldots, \ell_{j-1}, \ell_{j}^{\prime}$ be the sequence associated with $x$. Let $\mathrm{CFL}\left(\ell_{i}^{\prime \prime}\right)=\left(g_{1}, \ldots, g_{k^{\prime \prime}}\right)$ and $\mathrm{CFL}\left(\ell_{j}^{\prime}\right)=\left(g_{1}^{\prime}, \ldots, g_{k^{\prime}}^{\prime}\right)$ We have

$$
\operatorname{CFL}(x)=\left(g_{1}, \ldots, g_{k^{\prime \prime}}, \ell_{i+1}, \ldots, \ell_{j-1}, g_{1}^{\prime}, \ldots, g_{k^{\prime}}^{\prime}\right)
$$

where it is understood that if $\ell_{i}^{\prime \prime}=1$ (resp. $\ell_{j}^{\prime}=1$ ), then the first $k^{\prime \prime}$ terms (resp. last $k^{\prime}$ terms) in $\mathrm{CFL}(x)$ vanish.

Let $x, y, z, w, w^{\prime} \in \Sigma^{+}$. Lemma 5 gives relations between the Lyndon factorizations of two overlapping words $w, w^{\prime}$, i.e., such that $w=x y, w^{\prime}=y z$, and the Lyndon factorization of the overlap $y$, when $y$ is non-simple (as a suffix of $w$ and as a prefix of $w^{\prime}$ ). Indeed observe that both $w$ and $w^{\prime}$ are substrings of the same word $x y z$. As a consequence of Lemma 5 , the words $w, w^{\prime}$ may share common Lyndon factors between them and with $x y z$. Moreover, some of these factors may be in $y$. More precisely, let $\operatorname{CFL}(w)=\left(\ell_{1}, \ldots, \ell_{k}\right)$ and $\operatorname{CFL}\left(w^{\prime}\right)=\left(f_{1}, f_{2}, \ldots, f_{h}\right)$. If $y$ is a non-simple suffix of $w$ and a non-simple prefix of $w^{\prime}$, then there are indexes $i, j$, with $1 \leq i<k, 1<j \leq h$, such that $y=\ell_{i}^{\prime \prime} \ell_{i+1} \cdots \ell_{k}=f_{1} \cdots f_{j-1} f_{j}^{\prime}$, where $\ell_{i}^{\prime \prime}$ is a suffix of $\ell_{i}$ and $f_{j}^{\prime}$ is a prefix of $f_{j}$. Let $\operatorname{CFL}\left(\ell_{i}^{\prime \prime}\right)=\left(g_{1}, \ldots, g_{k^{\prime \prime}}\right)$ and $\operatorname{CFL}\left(f_{j}^{\prime}\right)=\left(g_{1}^{\prime}, \ldots, g_{k^{\prime}}^{\prime}\right)$. By Lemma 5 we have $\operatorname{CFL}(y)=\left(g_{1}, \ldots, g_{k^{\prime \prime}}, \ell_{i+1}, \ldots, \ell_{k}\right)=\left(f_{1}, \ldots, f_{j-1}, g_{1}^{\prime}, \ldots, g_{k^{\prime}}^{\prime}\right)$. Since the Lyndon factorization can be computed in linear time, the above result could lead to efficient measures of similarities between words. These measures could be used to capture words that may be overlapping.

\section{Conclusions and Open Problems}

In this paper we investigate new properties of the Lyndon factorization and of the canonical inverse Lyndon factorization, aimed to answer to string queries 
by using these factorizations. Our main result, Proposition 6, gives an upper bound on the length of the longest common prefix of two factors of a word and this upper bound has relationships with the factors in ICFL. This result could also be applied to investigate parallel approaches to sorting suffixes of a word $w$ with a nontrivial inverse Lyndon factorization. Indeed, the above mentioned bound could relate sorting suffixes of $w$ to sorting factors of $w$ of bounded length. In addition, we state a property showing that substrings of the same word could share common factors of the Lyndon factorization (Lemma 5). This property could be extended to two words that share a common overlap to capture the suffix-prefix relationship between them. It is an open problem if Lemma 5 extends to $\operatorname{ICFL}(w)$. This extension, if it exists, may be of interest in the well known problem of efficient computation of the suffix-prefix relationship. This is an interesting problem in the analysis of sequencing data $[7,8]$ and in the construction of overlap graphs for a collection of strings. We believe that the above results could shed new light in further applications of the Lyndon and the inverse Lyndon factorization and this is the goal of our future research work.

Acknowledgments. The authors thank the anonymous referees for their helpful suggestions.

\section{References}

1. Apostolico, A., Crochemore, M.: Fast parallel Lyndon factorization with applications. Math. Syst. Theory 28(2), 89-108 (1995)

2. Bannai, H., Tomohiro, I., Inenaga, S., Nakashima, Y., Takeda, M., Tsuruta, K.: The "Runs" theorem. SIAM J. Comput. 46(5), 1501-1514 (2017)

3. Bannai, H., Tomohiro, I., Inenaga, S., Nakashima, Y., Takeda, M., Tsuruta, K.: A new characterization of maximal repetitions by Lyndon trees. In: Proceedings of the Twenty-Sixth Annual ACM-SIAM Symposium on Discrete Algorithms, SODA 2015, San Diego, CA, USA, 4-6 January 2015, pp. 562-571 (2015)

4. Berstel, J., Perrin, D., Reutenauer, C.: Codes and Automata. Encyclopedia of Mathematics and its Applications, vol. 129. Cambridge University Press, Cambridge (2009)

5. Bonizzoni, P., De Felice, C., Zaccagnino, R., Zizza, R.: Inverse Lyndon words and inverse Lyndon factorizations of words. Adv. Appl. Math. 101, 281-319 (2018)

6. Bonizzoni, P., De Felice, C., Zaccagnino, R., Zizza, R.: Lyndon words versus inverse Lyndon words: queries on suffixes and bordered words. CoRR abs/1911.01851 (2019). http://arxiv.org/abs/1911.01851

7. Bonizzoni, P., Della Vedova, G., Pirola, Y., Previtali, M., Rizzi, R.: An externalmemory algorithm for string graph construction. Algorithmica 78(2), 394-424 (2017)

8. Bonizzoni, P., Della Vedova, G., Pirola, Y., Previtali, M., Rizzi, R.: FSG: fast string graph construction for de novo assembly. J. Comput. Biol. 24(10), 953-968 (2017)

9. Chen, K.T., Fox, R.H., Lyndon, R.C.: Free differential calculus, IV. The quotient groups of the lower central series. Ann. Math. 68, 81-95 (1958)

10. Choffrut, C., Karhumäki, J.: Combinatorics of words. In: Rozenberg, G., Salomaa, A. (eds.) Handbook of Formal Languages, pp. 329-438. Springer, Heidelberg (1997). https://doi.org/10.1007/978-3-642-59136-5_6 
11. Crochemore, M., Désarménien, J., Perrin, D.: A note on the Burrows-Wheeler transformation. Theoret. Comput. Sci. 332(1), 567-572 (2005)

12. Crochemore, M., Hancart, C., Lecroq, T.: Algorithms on Strings. Cambridge University Press, Cambridge (2007)

13. Daykin, J.W., Iliopoulos, C.S., Smyth, W.F.: Parallel RAM algorithms for factorizing words. Theor. Comput. Sci. 127(1), 53-67 (1994)

14. Dolce, F., Restivo, A., Reutenauer, C.: On generalized Lyndon words. Theor. Comput. Sci. 777, 232-242 (2019)

15. Duval, J.: Factorizing words over an ordered alphabet. J. Algorithms 4(4), 363-381 (1983)

16. Fredricksen, H., Maiorana, J.: Necklaces of beads in $k$ colors and $k$-ary de Brujin sequences. Discrete Math. 23(3), 207-210 (1978)

17. Ghuman, S.S., Giaquinta, E., Tarhio, J.: Alternative algorithms for Lyndon factorization. In: Proceedings of the Prague Stringology Conference 2014, Prague, Czech Republic, 1-3 September 2014, pp. 169-178 (2014)

18. Kärkkäinen, J., Kempa, D., Nakashima, Y., Puglisi, S.J., Shur, A.M.: On the size of Lempel-Ziv and Lyndon factorizations. In: 34th Symposium on Theoretical Aspects of Computer Science, STACS 2017, Hannover, Germany, 8-11 March 2017, pp. 45:1-45:13 (2017)

19. Kufleitner, M.: On bijective variants of the Burrows-Wheeler transform. In: Proceedings of the Prague Stringology Conference 2009, Prague, Czech Republic, 31 August-2 September 2009, pp. 65-79 (2009)

20. Lothaire, M.: Algebraic Combinatorics on Words. Encyclopedia of Mathematics and its Applications, vol. 90. Cambridge University Press, Cambridge (1997)

21. Lothaire, M.: Combinatorics on Words. Cambridge University Press, Cambridge (1997)

22. Lothaire, M.: Applied Combinatorics on Words. Cambridge University Press, Cambridge (2005)

23. Mantaci, S., Restivo, A., Rosone, G., Sciortino, M.: An extension of the BurrowsWheeler transform. Theor. Comput. Sci. 387(3), 298-312 (2007)

24. Mantaci, S., Restivo, A., Rosone, G., Sciortino, M.: Sorting suffixes of a text via its Lyndon factorization. In: Proceedings of the Prague Stringology Conference 2013, Prague, Czech Republic, 2-4 September 2013, pp. 119-127 (2013)

25. Mantaci, S., Restivo, A., Rosone, G., Sciortino, M.: Suffix array and Lyndon factorization of a text. J. Discrete Algorithms 28, 2-8 (2014)

26. Mucha, M.: Lyndon words and short superstrings. In: Proceedings of the TwentyFourth Annual ACM-SIAM Symposium on Discrete Algorithms, SODA 2013, New Orleans, Louisiana, USA, 6-8 January 2013, pp. 958-972 (2013)

27. Reutenauer, C.: Free lie algebras. Oxford University Press (1993)

28. Urabe, Y., Kempa, D., Nakashima, Y., Inenaga, S., Bannai, H., Takeda, M.: On the size of overlapping Lempel-Ziv and Lyndon factorizations. In: 30th Annual Symposium on Combinatorial Pattern Matching, CPM 2019, 18-20 June 2019, Pisa, Italy. LIPIcs, vol. 128, pp. 29:1-29:11. Schloss Dagstuhl - Leibniz-Zentrum fuer Informatik (2019) 